\title{
Measurement of Face-touching Frequency in a Simulated Train
}

\author{
Kentaro Morita ${ }^{1, *}$, Kaho Hashimoto ${ }^{1}$, Masayuki Ogata $^{1}$, Hitomi Tsutsumi ${ }^{2}$, Shin-ichi Tanabe ${ }^{1}$, and Satoshi Hori ${ }^{3}$ \\ ${ }^{1}$ Waseda University, 169-8555 Shinjuku-ku, Tokyo, Japan \\ ${ }^{2}$ Showa Women's University, 154-8533 Setagaya-ku, Tokyo, Japan \\ ${ }^{3}$ Juntendo University, 113-8421 Bunkyo-ku, Tokyo, Japan
}

\begin{abstract}
Contact behavior in a public space influences the risk of contact infection because public spaces have many environmental surfaces contaminated with pathogens. It is useful for risk reduction to examine the factor of infection risk among behaviors. In the present study, a video monitoring survey was conducted in a simulated cabin of a commuter train, we had built, to investigate the relationship between face-touching frequencies and individual attributes. As a result, the average face-touching frequency was 17.8 times per hour. Of all face touches, mucosal contact was $42.2 \%$. Focusing on the sex, the face-touching frequency was significantly higher for the males than for the females. Focusing on the skin condition, the face-touching frequency of those who did not wear makeup was significantly higher than that of those who did. The significant sex differences may depend on the makeup. Focusing on pollution awareness, higher pollution awareness related to lower frequency. Thus, by improving pollution awareness of the environmental surfaces in public spaces, it is possible to reduce effectively face-touching frequency and, hence, infection risk.
\end{abstract}

\section{Introduction}

When infectious diseases are prevalent, the health of infected people is impaired, and individuals' quality of life declines [1]. Infectious disease control is important for people's healthy and comfortable lives. It will also result in reduced economic losses that are due to the burden of medical expenses and high absenteeism [2].

In indirect contact infection, one type of contact infection, the infection route is established by touching one's own facial mucosa with fingers that touched shared items exposed to coughing of infected people [3]. In public spaces it is useful to investigate the infection route by contact of fingers with the face because many unspecified people share many environmental surfaces.

A method to measure the number of face contacts has been used to grasp the process by which pathogens are brought into the body by contact with the fingers [4-7]. However, there are large variations in the number of face contacts depending on the measuring method and conditions, and few studies have studied the influence on contact behavior that are due to the difference in conditions. To cope with contact infection, the factors that affect contact behavior need to be determined.

In this research, similar to Hashimoto et al. [7], the frequency of face touching in a simulated train was measured and compared between different conditions, such as individual attributes. We aimed to evaluate the contact infection risk in public spaces by quantitatively analyzing daily contact behavior. Moreover, this study sought to establish a method for identifying the factors that increase the risk of contact infection caused by face contact and to reduce the major factors of involvement.

\section{Methods}

\subsection{Outline}

From September 27 to October 19, 2017, the experiment was conducted 12 times in the simulated cabin of a commuter train built in the experimental room. A total of 40 students (20 males) participated as subjects.

The study assumed that there is an unlimited number of people in the same space on a train and there is a high possibility that the infection will expand through shared items, such as straps. Moreover, as many smartphones are not regularly cleaned [8], there is a possibility that they are contaminated as well as shared items. Thus, the facetouching frequency was measured while subjects were using their smartphone in the simulated train.

In this experiment, sex, behavior content, posture, and personal characteristics, such as skin condition and pollution awareness, were taken into account to clarify the factors influencing face-touching frequency.

\subsection{Conditions}

As posture condition, sedentary and standing positions were set, and participants consistently adopted one posture during measurement. The positions were not mixed, and experiments were conducted individually.

As behavior content condition, face-touching frequency was measured while subjects were using their smartphone in the simulated train. For comparison, another condition was set where subjects were not allowed to use smartphones and did nothing.

\footnotetext{
* Corresponding author: morita@tanabe.arch.waseda.ac.jp
} 


\subsection{Simulated train}

Figure 1 shows the elevation and plan of the simulated train. Figure 2 shows the interior of the simulated train. With reference to the general dimensions of a train $[9,10]$, the cabin interior of a commuter train was reproduced. As reading advertisements under the doing-nothing condition is assumed, printed matter simulating advertisement was posted in the simulated train.

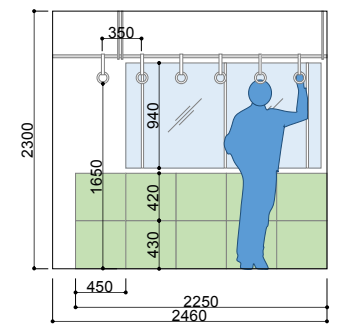

a) Elevation $[\mathrm{mm}]$

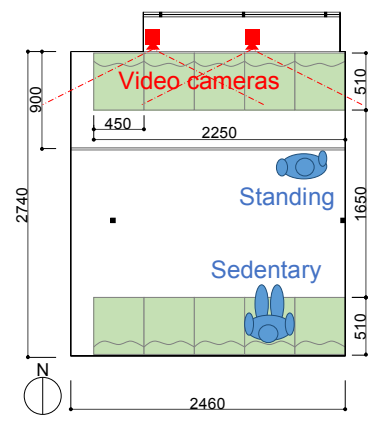

b) Plan $[\mathrm{mm}]$
Fig. 1. Elevation and plan of the simulated train (non-scale)

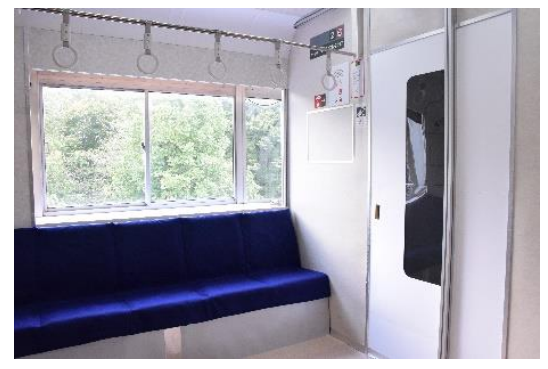

Fig. 2. Interior of the simulated train

\subsection{Measurement of face-touching frequency}

Face-touching frequency in the simulated train was measured by video monitoring. Upon shooting, the video camera was covered inconspicuously so that the examinees were not overly aware of being filmed.

Figure 3 shows the classification of the face-touching area. Contact frequency was measured by classifying the part on the face (mucous membrane of eyes, nose, mouth, and non-mucosal surface) contacted by any part of the fingers, palm, and back of the hand. Regarding the nose, only the lower part was included in the mucous membrane. For measurement, we counted only when the hand and the face are in direct contact with each other. Intermittent contacts were counted as one face touch until the fingers clearly separated from the face or the way of contact changed significantly.

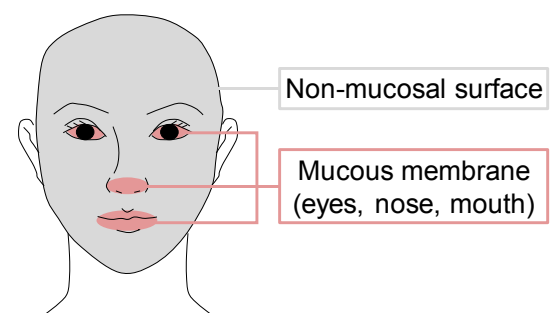

Fig. 3. Classification of the face-touching area

\subsection{Procedure}

Prior to the start of the experiment, the contents of the filming were explained to the participants. At the time of explanation, to avoid changes in face-touching behavior owing to well-known measurement contents, the participants were only told they would be filmed to show how they spend their time in a train. For each condition, the experimental time was set to 30 minutes. Two experiments were conducted consecutively, with a fiveminute interval. After completing the two experiments, we explained the measurement contents and conducted a questionnaire survey.

\subsection{Questionnaire survey}

Table 1 shows the questionnaire items. The section concerning skin surface was aimed at grasping the participants' skin conditions, such as the dry feeling of the skin surface, itching sensation, and presence or absence of use of glasses and contact lenses, which possibly influence face-touching frequency. In addition, taking into consideration the women participants, we also investigated the presence of makeup, which is believed to affect the skin condition of the face. The section on pollution awareness aimed to investigate awareness of environmental surfaces contamination and hand hygiene habits. In this study, as we are conducting experiments on smartphone usage inside the train, we investigated the awareness of contamination mainly with straps or handrails in the train and smartphones.

Table 1. Questionnaire items

\begin{tabular}{l|l}
\hline \multicolumn{2}{l}{ On skin surface } \\
\hline \hline$\# 1$ & Do you have atopic dermatitis? \\
\hline$\# 2$ & Do you have dry, sensitive skin? \\
\hline$\# 3$ & Do you have itching on your face? If yes, why? \\
\hline$\# 4$ & Do you usually wear makeup? \\
\hline$\# 5$ & $\begin{array}{l}\text { Do you mind when your makeup sticks to your hand when } \\
\text { touching your face? }\end{array}$ \\
\hline$\# 6$ & Do you wear glasses or contact lenses? \\
\hline On pollution awareness \\
\hline$\# 7$ & Do you feel resistive to hold the straps or handrails on trains? \\
\multirow{2}{*}{$\# 8$} & Do you usually hold the straps or handrails on trains? \\
\cline { 2 - 2 } & $\begin{array}{l}\text { [Always, Occasionally, Only when the train shakes strongly, } \\
\text { Never] }\end{array}$ \\
\cline { 2 - 2 } When and how often do you wash your hands? & $\begin{array}{l}\text { When - [After getting off the train, Before meals, After using } \\
\text { the toilet, After coming back home, Other] }\end{array}$ \\
\cline { 2 - 2 } & $\begin{array}{l}\text { How often - [Always, Often, Occasionally, Only when a hand } \\
\text { gets dirty, Never] }\end{array}$ \\
\hline \multirow{2}{*}{ D10 } & $\begin{array}{l}\text { Do you have a habit of disinfecting your smartphone with } \\
\text { alcohol? }\end{array}$ \\
\hline
\end{tabular}

\section{Results}

The calculation of face-touching frequency excluded sleep, observed in more than half the measurement time, and symptoms of rhinitis. In addition, one male participant rested his cheek in his hand during the entirety of the measurement time, and remarkably high facetouching frequency was shown; this participant was excluded from the subsequent significant difference test. IBM SPSS Statistics ver. 24 was used in the analysis. 


\subsection{Face-touching frequency}

\subsubsection{Comparison by each condition}

Figure 4 shows the differences in face-touching frequencies between each condition. The average facetouching frequency was 17.8 times per hour (median: 12.0 times/h) for all subjects. The average frequency of contact with facial mucous membrane was 8.0 times per hour (median: 4.0 times/h). When comparing behavior contents, face-touching frequency was higher for the doing-nothing than for the smartphone usage condition. However, verification by Welch's test showed no significant difference. In addition, no significant difference was observed even in the comparison between posture conditions. When compared by sex, face-touching frequency was higher for the males than for the females, and the significant difference was confirmed by Welch's test $(p<0.01)$.

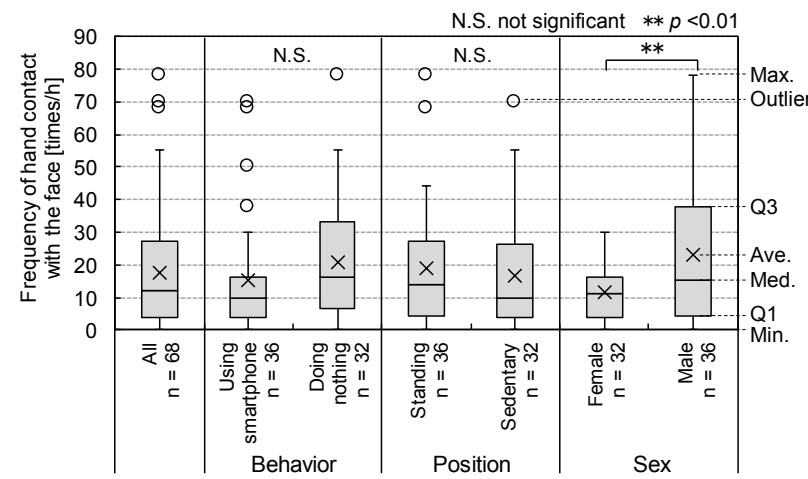

Fig. 4. Differences in face-touching frequencies between each condition

\subsubsection{Trend of frequency of face touching and contact with facial mucous membrane}

Figure 5 shows the trend of frequency of face touching and contact with facial mucous membrane. With increased face-touching frequency, the frequency of contact with the facial mucous membrane increased. Of all face touches, $42.2 \%$ involved contact with the mucous membrane surface, and $57.8 \%$, with the non-mucosal surface. The result of the mucosal contact ratio was similar to the past studies $[6,7]$.

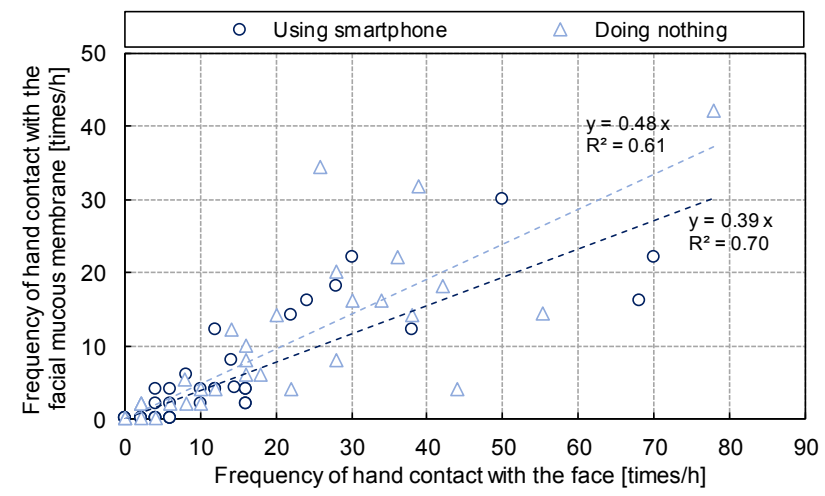

Fig. 5. Trend of frequency of face touching and contact with facial mucous membrane

\subsection{Relationship between the questionnaire survey result and face-touching frequency}

\subsubsection{Questionnaire on skin surface}

Figure 6 shows the differences in face-touching frequencies between skin conditions. When paying attention to the presence or absence of makeup, the average face-touching frequency was 12.4 times per hour (median: 12.0 times/h) for the participants who usually wear makeup, and 22.0 times per hour (median: 14.2 times/h) for those who do not. Welch's test showed that the frequency of face touching and contact with facial mucous membrane of those who do not wear makeup was significantly higher compared with those who do ( $p$ $<0.05$ ). Only two women were among the subjects who replied that they did not wear makeup. Significant sex differences described in 3.1.1 may depend on the presence or absence of makeup.

On the presence or absence of dry, sensitive skin, the average face-touching frequency was 10.9 times per hour (median: 8.0 times/h) for participants who indicated having dry, sensitive skin, and 19.7 times per hour (median: 14.5 times/h) for those who did not. Welch's test showed that the face-touching frequency of those who did not have dry, sensitive skin was significantly higher than that of those who did $(p<0.05)$. This trend was similar for the frequency of contact with facial mucous membrane, but no significant difference was observed.
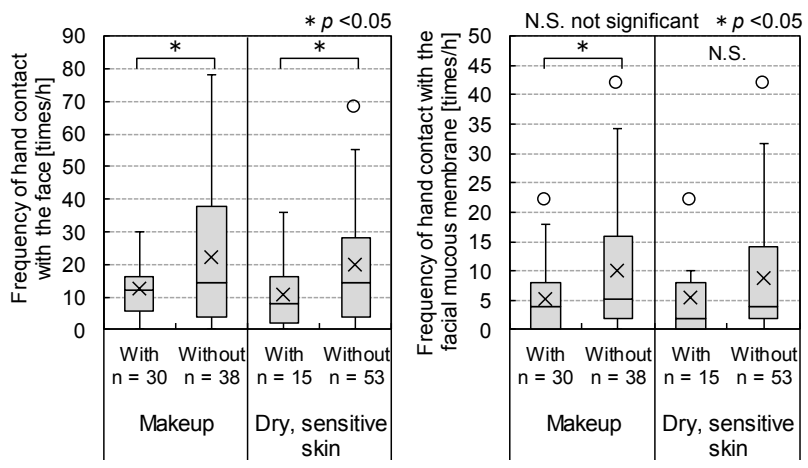

a) Face contact

b) Mucous membrane contact

Fig. 6. Differences in face-touching frequencies between skin conditions

\subsubsection{Questionnaire on pollution awareness}

Figure 7 shows the differences in face-touching frequencies between pollution awareness. Spearman's rank correlation coefficient $r_{s}$ was calculated to determine the relationship between face-touching behavior and questionnaire survey results on pollution awareness.

Participants who answered "always" for holding the straps on trains had higher face-touching frequencies than those who answered "occasionally" or "only when the train shakes strongly." A significant correlation was observed between face-touching frequency and habits of holding straps or handrails on trains $(p<0.01)$. This trend was similar for the contact with facial mucous membrane, for which a significant difference was observed $(p<0.05)$. 
Regarding the frequency of hand hygiene after getting off the train, participants who indicated not performing hand hygiene after getting off the train had higher facetouching frequencies than those who did, and a significant correlation was observed between frequency of contact with facial mucous membrane and frequency of hand hygiene after getting off the train $(p<0.05)$.

Further, there was a significant correlation between the habit of holding straps on trains and frequency of hand hygiene after getting off the train $\left(r_{s}=-0.354, p<0.01\right)$.
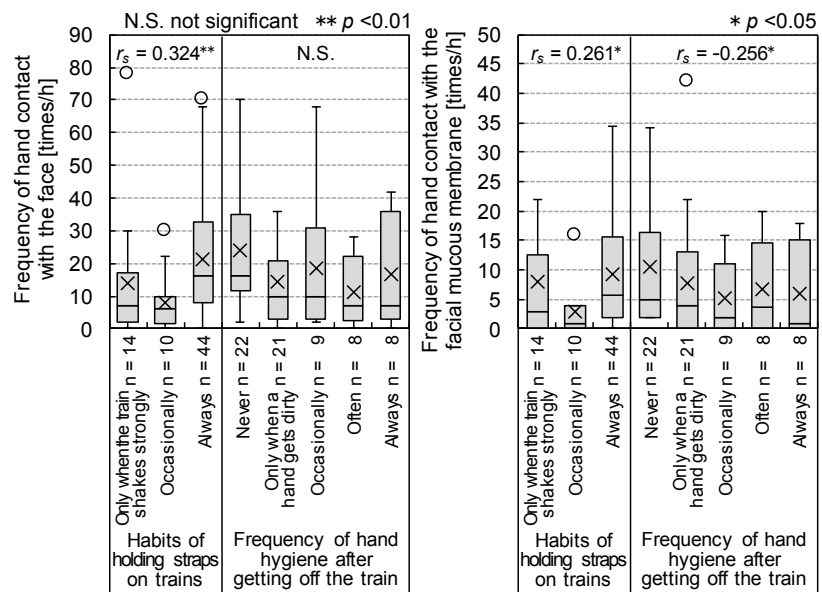

a) Face contact

b) Mucous membrane contact

Fig. 7. Differences in face-touching frequencies between pollution awareness

\section{Discussion}

With increased face-touching frequency, the frequency of contact with the facial mucous membrane increased. It can be said that an increase in face-touching frequency may increase the risk of infection.

Focusing on skin condition, the face-touching frequency of those who did not wear makeup was significantly higher than that of those who did. This trend was similar for the presence or absence of dry, sensitive skin. It is assumed that those who usually wear makeup may worry that their makeup would smudge owing to face touching, and therefore, they tend to avoid touching the face. Similarly, consciousness to avoid discomfort caused by face touching possibly worked on subjects who reported having dry, sensitive skin. From the above, there is a possibility that the face-touching frequency may be reduced in people with skin-related factors, such as makeup and skin symptoms that need to be considered.

Regarding pollution awareness, face-touching frequency was higher among participants characterized by a habit of holding straps in trains and low hand hygiene after getting off the train. This finding suggests that higher pollution awareness will lead to a lower face-touching frequency. If hand hygiene is not carried out after holding a strap, possibly causing contamination propagation, and if face-touching frequency is also high, the risk of contact infection will increase. Thus, by improving pollution awareness of the environmental surfaces in public spaces, it is possible to reduce effectively face-touching frequency and, hence, infection risk.

\section{Conclusion}

In this report, we measured and analyzed the frequency of face touching within a simulated train and showed factors that may affect face contact behavior. The findings obtained are shown below.

1) Of all face touches, mucosal contact was $42.2 \%$, and non-mucosal contact was $57.8 \%$, as in past studies $[6,7]$.

2) There were no clear trends or significant differences between the conditions of behavior content and posture and the frequency of face touching.

3) The frequency of face touching and contact with facial mucous membrane was higher for males than for females $(p<0.01)$. The difference may depend on the use of makeup.

4) The face-touching frequency of those who did not wear makeup was significantly higher than that of those who $\operatorname{did}(p<0.05)$.

5) The face-touching frequency of those who did not have dry, sensitive skin was significantly higher than that of those who did $(p<0.05)$.

6) The more that participants have a habit of holding the straps in trains and the less they perform hand hygiene after getting off the train, the higher the frequency of contact with facial mucous membrane.

This research is based on the collaborative research of Waseda University and Juntendo University. Experiments in this study were conducted in accordance with the provisions of the Waseda University Ethics Review Committee, and students from Waseda University cooperated as subjects. Part of this research was funded by the Young Scientists' Community (Early Bird Program), Waseda Research Institute for Science and Engineering.

\section{References}

1. J. Vietri, G. Prajapati, A.C. El Khoury, BMC Gastroenterol. 13, 16 (2013)

2. T.J. Bramley, D. Lerner, M. Sames, J. Occup. Environ. Med. 44, 822-829 (2002)

3. U.S. Department of Health and Human Services, HHS Pandemic Influenza Plan, Supplement 4 Infection Control (2005)

4. M. Nicas, D. Best, J. Occup. Environ. Hyg. 5, $347-$ 352 (2008)

5. J.D. Johnston, D. Eggett, M.J. Johnson, J.C. Reading, J. Occup. Environ. Hyg. 11, 625-632 (2014)

6. Y.L.A. Kwok, J. Gralton, M.L. McLaws, Am. J. Infect. Control. 43, 112-114 (2015)

7. K. Hashimoto, M. Ichikawa, Y. Akiyama, M. Ogata, S. Tanabe, S. Hori, H. Tsutsumi, Proc. Indoor Air 2018, 283 (flash drive format, 2018)

8. I. Morioka, K. Uda, M. Yamamoto, Jpn. J. Hyg. 70, 242-248 (2015)

9. T. Yoshikawa, Jpn. Soc. Mech. Eng. 111, 470-471 (2008)

10. A. Saito, H. Suzuki, H. Shiroto, K. Fujinami, H. Endo, S. Matsuoka, T. Hirai, K. Saito, Jpn. J. Ergon. 42, 921 (2006) 